\title{
ATIVIDADE ACARICIDA DE Nicotiana tabacum SOBRE OVOS DE Rhipicephalus (Boophilus) microplus
}

\author{
[Acaricidal activity of Nicotiana tabacum against Rhipicephalus (Boophilus) microplus eggs]
}

\author{
Izabela Mesquita Araújo', Mateus Borges ${ }^{1}$, Nailde de Paula Silva1, Hêmina Carla Vilela ${ }^{2}$, Rodrigo \\ Martins Fráguas $^{3}$, Isis Abel ${ }^{1^{*}}$ \\ ${ }^{1}$ Laboratório de Epidemiologia e Geoprocessamento, Instituto de Medicina Veterinária, Universidade Federal do Pará, \\ Castanhal, Pará, Brasil. \\ ${ }^{2}$ Centro Universitário de Lavras. \\ ${ }^{3}$ Laboratório de Química, Faculdade da Amazônia, FAMA, Vilhena, Rondônia, Brasil.
}

RESUMO - A utilização de acaricidas sintéticos é a forma mais comum para o controle do carrapato-do-boi, Rhipicephalus (Boophilus) microplus. Porém, os problemas acarretados pelo o uso indiscriminado desses produtos têm impulsionado a busca por métodos alternativos como a utilização de compostos a base de plantas medicinais. Nesse contexto, este estudo objetivou avaliar a eficácia de diferentes métodos de extração do fumo de corda (Nicotiana tabacum) contra ovos de $R$. microplus. Para a obtenção dos extratos da planta, foram utilizadas as seguintes técnicas: maceração, destilação e extração de Soxhlet, todas utilizando a água como solvente. Os ovos foram organizados em alíquotas de $100 \mathrm{mg}$ para serem tratados com os extratos e, posteriormente, observados diariamente para o registro dos parâmetros relativos à eclosão larval. Observou-se um aumento significativo no período de incubação quando foram utilizados os extratos obtidos por maceração ou Soxhlet. O período e o percentual de eclosão foram significativamente reduzidos, com a utilização de qualquer uma das técnicas de extração. Portanto, o extrato aquoso de $N$. tabacum, sem a necessidade de formulações, é eficaz para impedir a eclodibilidade larval sob as condições empregadas nesta pesquisa.

Palavras-Chave: carrapato-do-boi; controle; fumo de rolo.

\begin{abstract}
The use of synthetic acaricides is the most common way to control the cattle tick, Rhipicephalus (Boophilus) microplus. However, problems that result from the indiscriminate use of these products have driven the search for alternative methods sucj as the use of compunds based on medicinal plants. This study aimed to evaluate the effectiveness of different extraction methods of rope tobacco (Nicotiana tabacum) against $R$. microplus eggs. In order to obtain bioactive compounds of the plant, the following techniques were employed: maceration, distillation and Soxhlet extraction, all of them using water as solvent. Eggs were organized in pools of $100 \mathrm{mg}$ to be treated with the extracts and, subsequently, they were observed daily for the record of larval hatching parameters. A significant increase in incubation period was observed when maceration or soxhlet extracts were used. Larval hatching period and hatching rate were significantly reduced, when any extraction technique was employed. Therefore, $N$. tabacum aqueous extract, without the need of formulations, is efficient to avoid larval hatchability under the conditions used in this research.
\end{abstract}

Keywords: cattle tick; control; rope tobacco.

\footnotetext{
* Autor para correspondência. E-mail: isisabel@ufpa.br Recebido: 13 de março de 2016.

Aceito para publicação: 26 de abril de 2016.
} 


\section{INTRODUÇÃO}

No cenário econômico, o carrapato Rhipicephalus (Boophilus) microplus (Canestrini, 1887) (Acari, Ixodidae) é importante devido aos grandes prejuízos ocasionados à pecuária. Tradicionalmente, o método de controle desse carrapato é baseado na utilização de acaricidas sintéticos. Porém, o uso indiscriminado desses produtos tem selecionado populações geneticamente resistentes (Andreotti et al., 2011). Nesse contexto, o estudo de alternativas ao controle, como o desenvolvimento de vacinas e a utilização de plantas medicinais, vem se intensificando nos últimos anos, inclusive no Brasil (Isman \& Grienensen, 2014).

Aproximadamente 55 espécies de plantas já foram testadas contra o R. microplus, incluindo Nicotiana tabacum L. (Solanales: Solanaceae) (Borges, Sousa, Barbosa, 2011). O efeito carrapaticida de $N$. tabacum sobre $R$. microplus já foi verificado predominantemente sobre fêmeas (Olivo et al., 2009; Rodríguez, Rodríguez, Cruz, 2010; Leal et al., 2014). Porém são necessários mais estudos, voltados principalmente para as diferentes formas de obtenção e aplicação dos extratos dessa planta (Olivo et al., 2009). No presente trabalho foi avaliado o efeito acaricida in vitro dos extratos aquosos das folhas curadas de $N$. tabacum sobre ovos de R. microplus.

\section{MATERIAL E MÉTODOS}

As folhas curadas de N. tabacum foram adquiridas em estabelecimento comercial no município de Lavras, estado de Minas Gerais, Brasil, em 2012, sendo sempre da mesma procedência. Para a obtenção dos extratos aquosos, as folhas foram pesadas e picadas manualmente. A extração ocorreu utilizando-se três métodos: refluxo, destilação simples e maceração (Azmir et al., 2013). Para cada método de extração, arbitrou-se a utilização de $50 \mathrm{~g}$ de folha picada e $100 \mathrm{~mL}$ de água deionizada, como solvente.

$\mathrm{Na}$ extração sob refluxo, a mistura contendo folha picada de $N$. tabacum e água deionizada foi submetida ao extrator de Soxhlet, após duas horas, o extrato aquoso foi retirado do aparelho e filtrado. $\mathrm{Na}$ técnica de destilação simples, o extrato da mistura foi obtido após três horas de contato com o solvente. Na maceração clássica, o material picado permaneceu em contato com o solvente por 24 horas.

Cerca de 200 fêmeas de carrapatos ingurgitadas foram manualmente colhidas de bovinos mestiços, isentos de carrapaticida químico há 40 dias, pertencentes a uma fazenda de gado de leite no município de Ribeirão Vermelho $\left(21^{\circ} 11^{\prime} 17.70^{\prime}\right.$ 'S 4503'50.82”O), sul de Minas Gerais, Brasil. Os carrapatos foram identificados como $R$. microplus de acordo com Barros-Battesti, Arzua \& Bechara (2006). Em seguida foram higienizados com hipoclorito a $1 \%$ e água destilada, secos em papel filtro, e dispostos em placas de Petri para realização da postura (Melo, Reis, Bittencourt, 2006). Os ovos obtidos foram organizados em alíquotas de $100 \mathrm{mg}$ e dispostos em tubos de ensaio vedados com algodão hidrófilo. Quatro grupos experimentais constituídos por 10 alíquotas de $100 \mathrm{mg}$ cada, foram montados: grupo I (controle), ovos tratados apenas com água deionizada; grupo II, ovos tratados com o extrato obtido por maceração; grupo III, ovos tratados com o extrato obtido sob refluxo; e grupo IV, ovos tratados com o extrato obtido por destilação simples.

Cada alíquota ficou submersa em $1 \mathrm{~mL}$ de solução dos extratos, teste ou controle, por cinco minutos, conforme Ribeiro et al. (2008). Em seguida, os tubos de ensaio foram invertidos para escoamento da solução e um novo algodão hidrófilo foi utilizado para vedar os tubos. Esses foram mantidos à temperatura ambiente e observados diariamente para o acompanhamento dos parâmetros biológicos relativos à eclosão larval de acordo com Melo, Reis \& Bittencourt (2006): período de incubação, período de eclosão e percentual de eclosão.

Foi aplicado o teste Shapiro-Wilk para verificar a normalidade dos dados de todos os tratamentos. Quando os dados não apresentavam distribuição normal, foi aplicado o teste Kruskal-Wallis. Caso contrário, a comparação das médias se deu com submissão dos dados à análise de variância (ANOVA), após a aplicação do teste de homogeneidade de variâncias. Quando verificados comportamentos distintos foi aplicado o teste de Dunnett T3, para identificar as diferenças entre os efeitos carrapaticidas dos tratamentos. Todos os testes foram aplicados com nível de significância de 5\%, utilizando-se o software SPSS v.20.0.

\section{RESULTADOS E DISCUSSÃO}

O período de incubação para os grupos II e III (34 dias) foi significativamente mais longo do que nos grupos I e IV ( 31 e 32 dias, respectivamente) (p < 0,05 ) (Tabela 1). A interferência no período de incubação dos ovos pode ser importante para o controle de $R$. microplus. Sendo um carrapato monoxeno, a fase não parasitária é o período mais curto do ciclo biológico, porém de mais exposição às condições ambientais. Sendo assim, quanto maior for o período de incubação, maior será o período em que os ovos estarão expostos às condições adversas do ambiente, o que pode levar à não eclosão (Sutherst \& Bourne, 2006). 
Tabela 1. Parâmetros relativos à eclosão larval de ovos de $R$. (B.) microplus submetidos a tratamentos com água deionizada e extratos aquosos de fumo de rolo.

\begin{tabular}{lccc}
\hline Grupos & Período de incubação (dias)* & Período de eclosão (dias)* & Percentual de eclosão (\%)* \\
\hline I (controle) & $31,50 \pm 0,71^{\mathrm{a}}$ & $8,60 \pm 1,71^{\mathrm{a}}$ & $76,00 \pm 14,30^{\mathrm{a}}$ \\
II (maceração) & $34,00 \pm 1,63^{\mathrm{b}, \mathrm{c}}$ & $2,00 \pm 1,33^{\mathrm{b}}$ & $14,00 \pm 5,16^{\mathrm{b}}$ \\
III (refluxo) & $34,30 \pm 1,49^{\mathrm{b}}$ & $1,20 \pm 0,63^{\mathrm{b}}$ & $11,00 \pm 3,16^{\mathrm{b}}$ \\
IV (destilação) & $32,00 \pm 0,82^{\mathrm{a}, \mathrm{c}}$ & $3,60 \pm 2,32^{\mathrm{b}}$ & $25,00 \pm 19,58^{\mathrm{b}}$ \\
\hline
\end{tabular}

*média \pm desvio padrão. Valores seguidos de letras diferentes em uma mesma coluna são estatisticamente diferentes $(\mathrm{p} \leq 0,05)$.

O período de eclosão dos grupos tratados com os extratos foi significativamente menor que o observado no grupo controle. É possível que esse parâmetro tenha sido influenciado pelo percentual de eclosão, que também foi significativamente menor entre os tratamentos $(\mathrm{p}<0,05)$, afinal um baixo percentual de eclosão pode ser concluído em menos tempo. O percentual de eclosão dos grupos II, III e IV foi de $14 \%, 11 \%$ e $25 \%$, respectivamente, enquanto que para o grupo I foi de $76 \%$. Já o período de eclosão para o grupo IV foi de 4 dias, enquanto os grupos II e III foram os que apresentaram o período mais curto, 2 dias e 1 dia, respectivamente, e o grupo I apresentou um período equivalente a 9 dias. Não houve diferença estatística entre os grupos tratados para ambas as variáveis, porém todos diferiram significativamente do grupo controle (Tabela 1).

Esse é o primeiro relato a respeito do efeito carrapaticida de $N$. tabacum sobre os ovos de $R$. microplus. Há estudos anteriores que empregam formulações diversas (extrato + cal ou detergente neutro ou glicerina) sobre fêmeas, com eficácia variável (Olivo et al., 2009; Leal et al., 2014). Porém, mesmo quando aplicado sobre fêmeas, os extratos parecem influenciar a biologia dos ovos e larvas oriundos das fêmeas tratadas (Leal et al., 2014). Nesse estudo, optou-se por padronizar a concentração dos extratos, porém diversificando os métodos de extração visando obter melhores resultados, como preconizado por Olivo et al. (2009). Embora esse estudo tenha empregado ovos ao invés das fêmeas utilizadas por Leal et al. (2014), não foi necessária a associação de outros elementos para se observar uma significativa interferência do fumo de corda sobre a eclodibilidade das larvas. Ainda, os extratos obtidos por diferentes métodos tiveram resultados semelhantes estatisticamente. Não foi necessário a uso de álcool na extração para se obter resultado satisfatório, contradizendo Nogueira \& Barci (2003) que acreditam que as extrações são mais eficientes na presença do álcool.

Os ovos dos grupos II e III apresentaram um aspecto ressecado e coloração escura. Barbosa et al. (2013) fizeram a mesma observação dos ovos de $R$. microplus tratados com o extrato de Guarea kunthiana. É possível que o extrato de N. tabacum tenha removido a cera de revestimento que protege os ovos contra a desidratação, o que possibilitou a troca osmótica dos ovos com o meio, e consequentemente o aparecimento desse aspecto ressecado e enrugado (Politi et al., 2015).

Avaliar a sobrevivência larval não foi um dos objetivos desse trabalho. Porém, observou-se que as larvas eclodidas oriundas de ovos tratados com extrato morreram poucos dias depois. O extrato de $N$. tabacum pode ter apresentado uma toxidade por contato. O resíduo do extrato presente nos tubos de ensaio em contato com as larvas poderia ter sido letal, causando a mortalidade (Ribeiro et al., 2008). Estudos apontam que $N$. tabacum possui atividade inseticida devido a presença de alcaloides como a nicotina, um inseticida de contato não persistente que age no sistema nervoso dos insetos (CastilloSánchez, Jiménez-Osornio, Delgado-Herrera, 2010). Diante disso, embora a ação acaricida da nicotina ainda não tenha sido estudada, sugere-se que essa atividade em $N$. tabacum esteja relacionada a esse metabólito, como ressaltado por Rodríguez, Rodríguez \& Cruz (2010), o que reforça a necessidade de mais estudos, visando principalmente o isolamento desses princípios ativos com ação acaricida. Ainda, experimentos mais aprofundados envolvendo histopatologia e histoquímica são necessários para observar as possíveis alterações histológicas provocadas pelo extrato aquoso de $N$. tabacum sobre ovos e larvas de $R$. microplus.

\section{CONCLUSÃO}

Nas condições do presente estudo, o extrato aquoso das folhas curadas de $N$. tabacum se apresentou como uma alternativa eficaz para inviabilizar o desenvolvimento de ovos de $R$. microplus, independentemente do método de extração utilizado.

\section{REFERÊNCIAS}

ANDREOTTI, R. et al. Acaricide resistance of Rhipicephalus (Boophilus) microplus in State of Mato Grosso do Sul, Brazil. Revista Brasileira de Parasitologia Veterinária, p. 127-133, 2011. 
AZMIR, J. et al. Techniques for extraction of bioactive compounds from plant materials: A review. Journal of Food Engineering, p.426-436, 2013.

BARBOSA, C. S. et al. In vitro activities of plant extracts from the Brazilian Cerrado and Pantanal against Rhipicephalus (Boophilus) microplus (Acari: Ixodidae). Experimental and Applied Acarology, v. 60, p. 421-430, 2013.

BARROS-BATTESTI, D. M.; ARZUA, M.; BECHARA, G. H. Carrapatos de Importância Médico-Veterinária da Região Neotropical: Um guia ilustrado para identificação de espécies. São Paulo: Vox/ICTTD-3/Butantan, 2006.

BORGES, L. M. F.; SOUSA, L. A. D.; BARBOSA, C. S. Perspectives for the use of plant extracts to control the cattle tick Rhipicephalus (Boophilus) microplus. Revista Brasileira de Parasitologia Veterinária, p. 89-96, 2011.

CASTILLO-SÁNCHEZ, L. E.; JIMÉNEZ-OSORNIO, J. J.; DELGADO-HERRERA, M. A. Secondary metabolites of the Annonaceae, Solanaceae and Meliaceae families used as biological control of insects. Tropical and Subtropical Agroecosystems, p. 445 -462, 2010.

ISMAN, M. B.; GRIENENISEN M. L. Botanical insecticide research: many publications, limited useful data. Trends Plant Science, 19 (3), 2014.

LEAL, G. S. et al. Atividade Carrapaticida do Fumo de Corda. In: Seminário de Iniciação Científica e Pós-Graduação da Embrapa Tabuleiros Costeiros, 4, 2014, Sergipe, Anais. Sergipe: Embrapa, 2014. p. 200-209.

MELO, D. R.; REIS, R. C. S.; BITTENCOURT, V. R. E. P. Patogenicidade in vitro do fungo Metarhizium anisopliae (Metschnikoff, 1878) Sorokin, 1883, sobre o carrapato Boophilus microplus (Canestrini, 1887). Revista Brasileira de Parasitologia Veterinária, v.14, n.4, p.157-162, 2006.

NOGUEIRA, A. H. C.; BARCI, L. A. G. Avaliação da atividade acaricida do fumo de corda associado à cal virgem no controle de larvas de Boophilus microplus em condições de laboratório. Arquivo do Instituto Biológico, v.70, p.3, 2003.

OLIVO, J. C. et al. Extrato aquoso de fumo em corda no controle do carrapato de bovinos. Ciência Rural, v.39, n.4, p. 1131-1135, 2009.

POLITI, F. A. S. et al. Action of ethanolic extract from aerial parts of Tagetes patula L. (Asteraceae) on hatchability and embryogenesis of Rhipicephalus sanguineus eggs (Acari: Ixodidae). Industrial Crops and Products, p. 55-61, 2015.

RIBEIRO, V. L. et al. Acaricidal activity of Calea serrata (Asteraceae) on Boophilus microplus and Rhipicephalus sanguineus. Veterinary Parasitology, p. 351-354, 2008.

RODRÍGUEZ, A.S; RODRÍGUEZ; C.; CRUZ, A. C. Efecto ixodicida de los extractos etanólicos de algunas plantas sobre garrapatas Rhipicephalus (Boophilus) microplus. Revista MVZ Córdoba, p. 2175-2184, 2010.

SUTHERST, R. W.; BOURNE, A. S. The effect of desiccation and low temperature on the viability of eggs and emerging larvae of the tick, Rhipicephalus (Boophilus) microplus (Canestrini) (Ixodidae). International Journal for Parasitology, p. 193-200, 2006. 